\title{
Polyamines are essential for virulence in Salmonella enterica serovar Gallinarum despite evolutionary decay of polyamine biosynthesis genes
}

Schroll, Casper; Christensen, Jens P.; Christensen, Henrik; Pors, Susanne E.; Thorndahl, Lotte; Jensen, Peter Ruhdal; Olsen, John E.; Jelsbak, Lotte

Published in:

Veterinary Microbiology

Link to article, DOI:

10.1016/j.vetmic.2014.01.034

Publication date:

2014

Document Version

Publisher's PDF, also known as Version of record

Link back to DTU Orbit

Citation (APA):

Schroll, C., Christensen, J. P., Christensen, H., Pors, S. E., Thorndahl, L., Jensen, P. R., Olsen, J. E., \& Jelsbak, L. (2014). Polyamines are essential for virulence in Salmonella enterica serovar Gallinarum despite evolutionary decay of polyamine biosynthesis genes. Veterinary Microbiology, 170(1-2), 144-150.

https://doi.org/10.1016/j.vetmic.2014.01.034

\section{General rights}

Copyright and moral rights for the publications made accessible in the public portal are retained by the authors and/or other copyright owners and it is a condition of accessing publications that users recognise and abide by the legal requirements associated with these rights.

- Users may download and print one copy of any publication from the public portal for the purpose of private study or research.

- You may not further distribute the material or use it for any profit-making activity or commercial gain

- You may freely distribute the URL identifying the publication in the public portal 


\title{
Polyamines are essential for virulence in Salmonella enterica serovar Gallinarum despite evolutionary decay of polyamine biosynthesis genes
}

\author{
Casper Schroll ${ }^{a}$, Jens P. Christensen ${ }^{a}$, Henrik Christensen ${ }^{a}$, Susanne E. Pors ${ }^{a}$, \\ Lotte Thorndahl ${ }^{\mathrm{a}}$, Peter R. Jensen ${ }^{\mathrm{b}}$, John E. Olsen ${ }^{\mathrm{a}}$, Lotte Jelsbak ${ }^{\mathrm{a}, *}$ \\ ${ }^{a}$ Department of Veterinary Disease Biology, Faculty of Life Sciences, University of Copenhagen, Frederiksberg C, Denmark \\ ${ }^{\mathrm{b}}$ Systems Biotechnology, Department of Systems Biology, Technical University of Denmark, Lyngby, Denmark
}

\section{A R T I C L E I N F O}

Article history:

Received 20 September 2013

Received in revised form 27 January 2014

Accepted 28 January 2014

\section{Keywords:}

Salmonella Gallinarum

Polyamines

Virulence

Host-specificity

Evolution

\begin{abstract}
A B S T R A C T
Serovars of Salmonella enterica exhibit different host-specificities where some have broad host-ranges and others, like $S$. Gallinarum and S. Typhi, are host-specific for poultry and humans, respectively. With the recent availability of whole genome sequences it has been reported that host-specificity coincides with accumulation of pseudogenes, indicating adaptation of host-restricted serovars to their narrow niches. Polyamines are small cationic amines and in Salmonella they can be synthesized through two alternative pathways directly from L-ornithine to putrescine and from L-arginine via agmatine to putrescine. The first pathway is not active in S. Gallinarum and S. Typhi, and this prompted us to investigate the importance of polyamines for virulence in $S$. Gallinarum. Bioinformatic analysis of all sequenced genomes of Salmonella revealed that pseudogene formation of the speC gene was exclusive for S. Typhi and S. Gallinarum and happened through independent events. The remaining polyamine biosynthesis pathway was found to be essential for oral infection with $S$. Gallinarum since single and double mutants in speB and speE, encoding the pathways from agmatine to putrescine and from putrescine to spermidine, were attenuated. In contrast, $s p e B$ was dispensable after intraperitoneal challenge, suggesting that putrescine was less important for the systemic phase of the disease. In support of this hypothesis, a $\Delta$ speE; $\Delta$ potCD mutant, unable to synthesize and import spermidine, but with retained ability to import and synthesize putrescine, was attenuated after intraperitoneal infection. We therefore conclude that polyamines are essential for virulence of $S$. Gallinarum. Furthermore, our results point to distinct roles for putrescine and spermidine during systemic infection.
\end{abstract}

(c) 2014 Elsevier B.V. All rights reserved.

\section{Introduction}

Salmonella enterica are Gram-negative facultative intracellular bacteria that infect a wide range of animal species as well as humans. The species $S$. enterica

\footnotetext{
* Corresponding author at: Department of Veterinary Disease Biology, Faculty of Life Sciences, University of Copenhagen, Stigbøjlen 4, DK-1870 Frederiksberg C, Denmark. Tel.: +45 3533 3752; fax: +45 35332757.

E-mail address: loj@sund.ku.dk (L. Jelsbak).
}

constitutes $>2500$ different serovars. Depending on the immune status of the infected host and the Salmonella serovar, diseases caused by $S$. enterica range from mild diarrhea to life threatening systemic infections. Some serovars like $S$. Typhimurium and $S$. Enteritidis mainly cause a self-limiting diarrhea and are able to cause disease in humans as well as a broad variety of animals. Other serovars like $S$. Gallinarum and $S$. Typhi are host restricted and only cause disease in chickens and humans, respectively. Upon ingestion of Salmonella contaminated food or water, the bacterium is able to pass the stomach and reach 
the intestines where a specific set of bacterial effector proteins are injected into the epithelial cells lining the wall of the small intestines thereby promoting invasion of these cells (Kaniga et al., 1995a,b). Following invasion of the epithelial cell layer Salmonella escapes to the underlying tissues (Knodler et al., 2010) where it is taken up by phagocytes like macrophages and dendritic cells, inside which Salmonella is able to survive and replicate, as reviewed in (Mastroeni and Grant, 2011). From here, it can rapidly spread through the lymphoid and blood systems to the spleen and liver resulting in a life-threatening systemic infection. Infections by host-restricted serovars are typically more severe with limited intestinal manifestation, often resulting in life threatening systemic diseases, indicating that host-adaptation has co-evolved with the ability to successfully pass the intestines and cause invasive typhoid-like systemic disease (Uzzau et al., 2001).

Recently, comparative genome analyses have revealed that host-restricted serovars diverged from non-restricted serovars through extensive genome reductions and accumulation of pseudogenes. Specifically, the genomes of S. Typhi and S. Gallinarum harbor 204 and 309 pseudogenes (Thomson et al., 2008), whereas their closest relatives among the broad host-range serovars $S$. Typhimurium and $S$. Enteritidis, only have 25 and 113 pseudogenes, respectively. This has fostered the hypothesis that genome decay has occurred during pathoadaptive evolution to the specific niches of highly virulent and hostrestricted Salmonella serovars (Thomson et al., 2008; Holt et al., 2009; Feng et al., 2013). In support of this hypothesis, $S$. Gallinarum and $S$. Typhi have lost several of the same metabolic pathways that are conserved in S. Typhimurium and $S$. Enteritidis, suggesting that the host-restricted serovars have similar nutrient resources in their respective intra-host niches (Parkhill et al., 2001; Thomson et al., 2008). As an example, in $S$. Typhimurium putrescine which together with spermidine are the most prominent polyamines in bacteria, can be synthesized via either of two redundant pathways: (1) from L-ornithine by the SpeC or

\section{A Putrescine/spermidine transport}

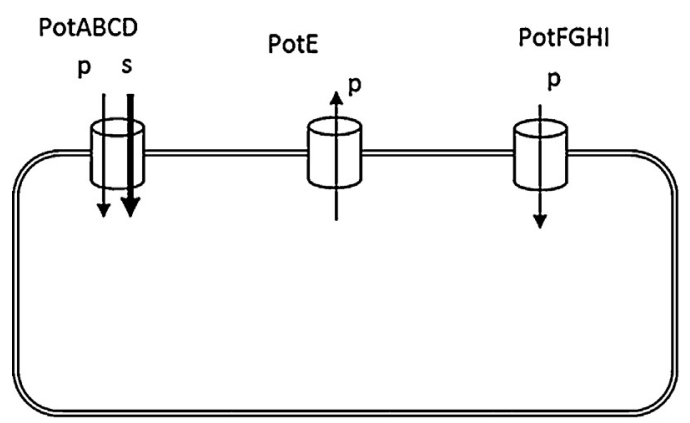

the acid-inducible SpeF ornithine decarboxylase (ODC) or (2) from L-arginine by SpeA and SpeB (Fig. 1). Spermidine is synthesized from putrescine and L-methionine by SpeE and SpeD. In both $S$. Gallinarum and $S$. Typhi, the polyamine biosynthesis gene speC encoding an ODC is a pseudogene (Thomson et al., 2008; Holt et al., 2009) and both serovars are classified as ODC negative. The content of polyamines, which represent small poly-cationic molecules present in almost all living cells, is controlled in bacteria by active transport across the membrane and biosynthesis (Igarashi and Kashiwagi, 2009). This indicates that polyamine biosynthesis could be dispensable in host-restricted serovars. As polyamine depletion is deleterious for virulence in S. Typhimurium (Jelsbak et al., 2012), these findings prompted us to investigate the role of polyamines in systemic infections of a host-restricted serovar of $S$. enterica. As a model we chose $S$. Gallinarum fowl typhoid infections, a disease highly similar to human typhoid caused by $S$. Typhi. Our results show that polyamines are essential for virulence in $S$. Gallinarum. These results have implications for development of novel treatment regimens for fowl typhoid aimed at inhibiting these enzymes, but also point to a conserved essentiality for polyamines in virulence of both host-restricted and non-restricted serovars of $S$. enterica. Furthermore, both $S$. Gallinarum and $S$. Typhi have lost one of two putrescine biosynthesis pathways supporting the hypothesis that part of the genome reduction observed in host-restricted serovars could be a result of deterioration of redundant metabolic pathways of the notoriously metabolically robust and broad host-range serovars $S$. Typhimurium and $S$. Enteritidis.

\section{Materials and methods}

\subsection{Bacterial strains and growth conditions}

Bacterial strains and plasmids are listed in Table 1. S. Gallinarum G9 was used as wild-type strain in all experiments. This strain has been described previously

\section{B Putrescine/spermidine biosynthesis}

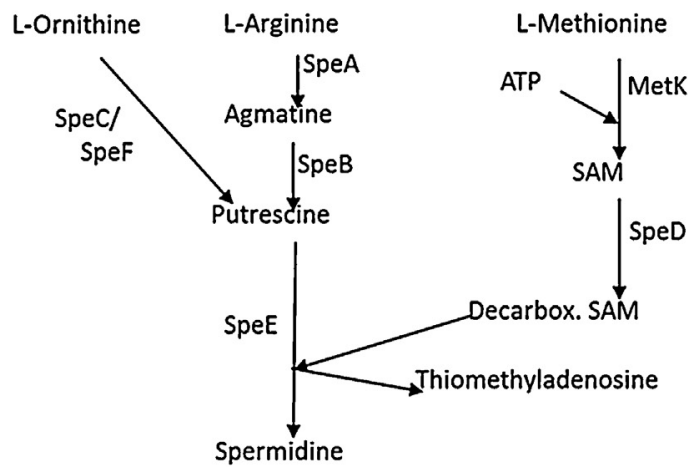

Fig. 1. Graphic presentation of putrescine/spermidine transporters and biosynthesis pathways. (A) A graphic presentation of the polyamine transporters with their substrate affinity indicated by $\mathrm{p}$ for putrescine and $\mathrm{s}$ for spermidine. (B) A graphic presentation of the polyamine biosynthesis pathways present in bacteria. SAM: S-adenosylmethionine. 
Table 1

Strains and plasmid used in the study.

\begin{tabular}{|c|c|c|}
\hline Strains & Relevant genotype & Reference \\
\hline S. Gallinarum G9 & Virulent reference strain & Jones et al. (2001) \\
\hline CAS87 & $\Delta$ speB & This work \\
\hline CAS89 & $\Delta s p e E$ & This work \\
\hline CAS95 & $\Delta$ speB; $\Delta$ speE & This work \\
\hline CAS103 & $\Delta$ speB; $\Delta$ speE/pLOJ318 & This work \\
\hline CAS114 & $\triangle$ potCD & This work \\
\hline CAS130 & $\Delta p o t C D ; \Delta$ speE & This work \\
\hline CAS159 & $\Delta$ potCD; $\Delta$ speE/pLOJ326 & This work \\
\hline \multicolumn{3}{|l|}{ Plasmids } \\
\hline pACYC177 & Cloning vector, $\mathrm{amp}^{\mathrm{R}}, \mathrm{kan}^{\mathrm{R}}$ & Chang and Cohen (1978) \\
\hline pKD46 & Plasmid with $\lambda$-Red recombinase expressed from arabinose inducible promoter & Datsenko and Wanner (2000) \\
\hline pKD3 & Template plasmid for $\lambda$-Red mutagenesis, cam $^{R}$ & Datsenko and Wanner (2000) \\
\hline pKD4 & Template plasmid for $\lambda$-Red mutagenesis, $\operatorname{kan}^{\mathrm{R}}$ & Datsenko and Wanner (2000) \\
\hline pCP20 & FLP plasmid for deletion of resistance cassette & Datsenko and Wanner (2000) \\
\hline pLOJ318 & pACYC177 expressing the $s p e B$ gene & Jelsbak et al. (2012) \\
\hline pLOJ326 & pACYC177 expressing the potCD genes & Jelsbak et al. (2012) \\
\hline
\end{tabular}

and its virulence is well defined (Jones et al., 2001; Chadfield et al., 2003). S. Gallinarum strains were maintained in LB media (Becton, Dickinson and Company). For solid medium, 1.5\% agar was added to give LB agar plates. Chloramphenicol $\left(15 \mu \mathrm{g} \mathrm{ml}^{-1}\right)$, kanamycin $\left(50 \mu \mathrm{g} \mathrm{ml}^{-1}\right)$, ampicillin $\left(100 \mu \mathrm{g} \mathrm{ml}^{-1}\right)$ or carbenicillin $\left(50 \mu \mathrm{g} \mathrm{ml}^{-1}\right)$ was added as required. Prior to all experiments the bacteria were grown for $20 \mathrm{~h}, 200 \mathrm{rpm}, 37^{\circ} \mathrm{C}$ in M9 minimal media (2 mM $\mathrm{MgSO}_{4}, 0.1 \mathrm{mM} \mathrm{CaCl} 2,0.4 \%$ glucose, $8.5 \mathrm{mM} \mathrm{NaCl}, 42 \mathrm{mM} \mathrm{Na} \mathrm{HPO}_{4}, 22 \mathrm{mM} \mathrm{KH}_{2} \mathrm{PO}_{4}$, $18.6 \mathrm{mM} \mathrm{NH}_{4} \mathrm{Cl}$ ) to deplete for carry-over polyamines. To complement for auxotrophy in S. Gallinarum G9 the M9 media was supplemented with $0.2 \mu \mathrm{g} \mathrm{ml}^{-1}$ thiamine, $0.2 \mu \mathrm{g} \mathrm{ml}^{-1}$ nicotinic acid, $25 \mu \mathrm{g} \mathrm{ml}^{-1}$ leucine, $5 \mu \mathrm{g} \mathrm{ml}^{-1}$ cystine and $5 \mu \mathrm{g} \mathrm{ml}^{-1}$ aspartic acid.

\subsection{Construction of strains}

Gene deletions and concomitant insertions of an antibiotic resistance cassette were constructed using Lambda Red mediated recombination as described elsewhere (Datsenko and Wanner, 2000). Double mutants were constructed by P22-mediated transductions after removing the antibiotic resistance cassette in the single mutant by FLP-mediated recombination with introduction of pCP20 (Datsenko and Wanner, 2000). All mutants were verified by PCR. Primers used to construct and verify mutants are listed in Table S1, supplementary material.

Supplementary material related to this article can be found, in the online version, at http://dx.doi.org/10.1016/ j.vetmic.2014.01.034.

\subsection{Sequence analysis of polyamine biosynthesis genes}

All 33 completed S. enterica genomes available in Genbank (Benson et al., 2012) were compared by use of BLAST (Altschul et al., 1997). Sequences of speC and speF homologs were downloaded and compared by multiple alignment with ClustalX (Larkin et al., 2007). Translation to protein was performed by EMBOSS (Rice et al., 2000).

\subsection{Infection of chickens}

Chicken challenge experiments were performed by competitive assays, where wild type and mutated strains were inoculated in equal amounts as previously described (Olsen et al., 2013). A few hours after hatching male Lohmann chickens were acclimatized in groups of five animals for three to five days. At all times they were supplied with food and water ad libitum. Three to five days old male Lohmann chickens were challenged in groups of five to ten animals with $10^{8}$ bacteria for oral challenge and $10^{6}$ for intraperitoneal challenge. Competitive indexes (C.I.) were calculated as previously described (Blondel et al., 2010; Jelsbak et al., 2012). The ratio between strains was confirmed by plate count method as described for spleen samples below. After four to five days (within a $24 \mathrm{~h}$ interval), the ratio between wild type and mutant strain was determined in the spleen, essentially as described by Jelsbak et al. (2012). In brief, dilutions of homogenized spleens were made in PBS and $100 \mu$ l aliquots were plated onto LB agar. Salmonella colonies were sub-cultured in parallel onto LB agar with and without the appropriate antibiotics to determine the ratio between wild type and the mutated strains in each animal. A mean competitive index was obtained from each group of individually challenged birds. A C.I. of 1.00 corresponded to wild type virulence. Chicken infection studies were performed with permission from the Danish Animal Experiments Inspectorate, license number 2009/561-1675.

\subsection{Infection of HD-11 chicken macrophages}

Uptake and survival in the chicken derived macrophage cell line HD-11 was measured using a gentamicin protection assay as previously described using a multiplicity of infection of 10:1 (Wallrodt et al., 2013), the only difference being the exchange of J774 cells with HD-11 cells.

\subsection{Statistical analysis}

C.I. outliers were identified by Grubbs' test. Comparison of mean ration of input and output pools of wild type and 
mutated bacteria in chicken infections were compared by Student $t$-test, and comparisons of CFU in macrophages was performed by ANOVA with Dunn's correction for multiple comparisons. All analysis was performed using GraphPad Prism6.

\section{Results and discussion}

\subsection{S. Typhi and S. Gallinarum have distinct mutations silencing the speC polyamine biosynthesis gene}

It has previously been reported that in both $S$. Gallinarum and S. Typhi the speC gene encoding ornithine decarboxylase is a pseudogene (Parkhill et al., 2001; Thomson et al., 2008), as a result of small out-of-frame deletions leading to premature stop codons. We reanalyzed the sequences and, interestingly, in S. Gallinarum there is a 4 base pair deletion after base pair 1268 in the reading frame whereas $S$. Typhi harbors an 11 base pair deletion after base pair 1130 (Table 2). This means that silencing of the speC genes in S. Typhi and S. Gallinarum occurred through independent events and indicates convergent evolution during adaptation to their respective niches, humans and poultry.

In contrast, examination of the spec locus of all Salmonella genomes in Genbank reveals that in all other serovars than $S$. Gallinarum and $S$. Typhi, the full-length speC gene is conserved with only few amino acid differences between strains (Table 2), indicating that silencing of speC in $S$. Gallinarum and $S$. Typhi is a relatively recent event occurring after their divergence from their respective ancestors. Interestingly, in $S$. Typhi, the speF gene encoding the alternative ODC is also a pseudogene, whereas in $S$. Gallinarum, while the speF gene is present, it is not functional as indicated by lack of ODC activity in this bacterium (Crichton and Old, 1990). Most other Salmonella strains show positive ODC reactions, indicating that only these highly host-restricted typhoid Salmonella strains S. Gallinarum and S. Typhi have lost the ability to convert ornithine into putrescine, perhaps reflecting similarities in their respective intrahost environments. Importantly, the alternative biosynthesis pathway to putrescine via SpeA and SpeB remains intact in all strains analyzed. This is highly indicative of an important role for putrescine biosynthesis in $S$. enterica and is supported by the fact that polyamines are essential for virulence in $S$. Typhimurium (Jelsbak et al., 2012). However, the parallel occurrence of loss-of-function of the ODC-pathway in the typhoid causing host-restricted serovars could reflect limited availability of substrates for putrescine biosynthesis in these serovars.

\subsection{Polyamines are essential for virulence in S. Gallinarum in orally infected chickens}

To analyze the role of polyamines in virulence of the host-restricted serovar, $S$. Gallinarum, individual deletion mutants of $s p e B$ and $s p e E$ were constructed. Deletion of $s p e B$ will abolish putrescine biosynthesis of the strain, as it depends on this pathway for the production of putrescine. The strain is still able to import putrescine from the environment via the PotFGHI transporter. It is likely that reduced putrescine biosynthesis will affect spermidine biosynthesis as well as putrescine is substrate for spermidine biosynthesis by SpeE. However, any imported putrescine will be available for conversion to spermidine by SpeE. Likewise, deletion of $s p e E$ will abolish spermidine biosynthesis of the strain, as it depends on this pathway for the production of spermidine. This could result in accumulation of putrescine, however, to counter this effect, the strain retains the ability to export excess putrescine via the putrescine exporter PotE. A double mutant of $s p e B$ and $s p e E(\Delta s p e B ; \Delta s p e E)$ was also constructed. This strain is deficient for both putrescine and spermidine biosynthesis, yet retains the ability to take up polyamines from the extracellular environment. Initially, the strains were tested for their ability to grow in minimal media without polyamines (M9). All mutants had growth rates similar to the $w t$, and when the strains were

Table 2

Comparison of the SpeC protein for all published genomes of Salmonella enterica subsp. enterica serotypes available in Genbank.

\begin{tabular}{|c|c|c|c|c|c|c|c|c|c|c|c|c|c|c|c|c|c|c|c|c|}
\hline $\begin{array}{l}\text { SpeC position } \\
\text { (Typhimurium) }\end{array}$ & 15 & 26 & 40 & 64 & 72 & 79 & 80 & 93 & 105 & 124 & 159 & 272 & 313 & 336 & 378 & 388 & 398 & 423 & 501 & 703 \\
\hline Typhimurium & $\mathrm{R}$ & $\mathrm{D}$ & $\mathrm{T}$ & A & V & V & G & $\mathrm{C}$ & G & Q & G & $\mathrm{S}$ & V & $\mathrm{D}$ & $\mathrm{N}$ & $\mathrm{P}$ & V & $\mathrm{R}$ & $\mathrm{R}$ & Q \\
\hline Enteritidis ATCC & & & & & & G & $\mathrm{E}$ & S & $\mathrm{D}$ & & & $\mathrm{L}$ & & & & & & & & \\
\hline Enteritidis & & & & & & & & $\mathrm{S}$ & $\mathrm{D}$ & & & $\mathrm{L}$ & & & & & & & Q & \\
\hline Dublin & $\mathrm{C}$ & & & & & G & & $\mathrm{S}$ & $\mathrm{D}$ & & & $\mathrm{L}$ & & & & & I & & Q & \\
\hline Heidelberg & & & & & & G & & & & & & & & & & & & & & \\
\hline Javiana & & & $\mathrm{P}$ & & & G & & & & & & & & & & & I & & $\mathrm{Q}$ & \\
\hline Schwarzengrund & & & & V & A & $\mathrm{G}$ & & & & & & & & & & & I & & Q & \\
\hline Choleraesuis & & & & & & G & & & $\mathrm{D}$ & & & & & & & & I & & Q & \\
\hline Paratyphi C & & & & & & G & & & $\mathrm{D}$ & & & & & & & & I & & Q & \\
\hline Weltevreden & & & & & & G & & & & & & $\mathrm{L}$ & & & & & I & & & \\
\hline Agona & & & & & & & & & & & & & & & & & I & & & \\
\hline Newport & & & & & & G & & & & & & $\mathrm{L}$ & G & $\mathrm{E}$ & & & & & & \\
\hline Paratyphi A & & $\mathrm{E}$ & & & & G & & & & & $\mathrm{S}$ & $\mathrm{P}$ & & & & & & & & $\mathrm{K}$ \\
\hline Paratyphi B & & & & & & G & & $\mathrm{S}$ & $\mathrm{D}$ & & & $\mathrm{L}$ & & & & & & & & \\
\hline Gallinarum & & & & & & $\mathrm{G}$ & & $\mathrm{S}$ & $\mathrm{D}$ & & & $\mathrm{L}$ & & & & $\mathrm{L}$ & & $\mathrm{K}^{\mathrm{a}}$ & & \\
\hline Typhi & & $\mathrm{E}$ & & & & G & & & & & & $\mathrm{P}$ & & & $\mathrm{H}^{\mathrm{b}}$ & & & & & \\
\hline
\end{tabular}

a From this position out of frame due to 4 bp deletion after bp 1268 resulting in stop codon at position 489 .

b From this position out of frame due to 11 bp deletion after bp 1130 resulting in stop codon at position 418 
Table 3

Competitive index analyses of $S$. Gallinarum mutants in oral infection of chickens.

\begin{tabular}{lll}
\hline Mutant vs wt & C.I \pm S.D. & Statistical analyses \\
\hline$\Delta$ speB & $0.14 \pm 0.19$ & Vs $1.0, p<0.0001$ \\
$\Delta$ speE & $0.23 \pm 0.2$ & Vs $1.0, p=0.0214$ \\
$\Delta$ speB; $\Delta$ speE & $0.03 \pm 0.03$ & Vs $1.0, p<0.0001$ \\
$\Delta$ speB; $\Delta$ speE/pACYC-speB & $0.77 \pm 1.05$ & Vs $\Delta$ speE, \\
& & not significant
\end{tabular}

Infections were performed as described in Section 2. Competitive indexes (C.I.) were calculated as described in Section 2. Standard deviation (S.D.) of the mean is indicated. Statistical analyses were performed as described in Section 2.

incubated for growth overnight they all reached the same stationary level of growth (Supplemental Figure 1).

Supplementary material related to this article can be found, in the online version, at http://dx.doi.org/10.1016/ j.vetmic.2014.01.034.

Next, the $\Delta$ speB-, $\Delta$ speE-single mutants and the $\Delta$ spe $; ; \Delta$ speE-double mutants were tested for their ability to pass the intestines and establish a systemic infection in chickens, as measured by bacterial counts in the spleen, after oral inoculations of equal amounts of wt and mutant bacteria as described by Blondel et al. (2010). Both the $\triangle$ spe $B$ and the $\triangle$ speE mutant had reduced Competitive indexes (C.I.) of $0.14 \pm 0.19$ and $0.23 \pm 0.2$ (Table 3), indicating that both putrescine and spermidine biosynthesis contribute to virulence. The double mutant had an even further reduction in virulence (C.I. $=0.03 \pm 0.03$ ) suggesting that polyamine biosynthesis is essential for oral infection of $S$. Gallinarum. Virulence could be partially restored by reintroducing the speB gene on a plasmid (C.I. $=0.77 \pm 1.05)$.

\subsection{Spermidine is the major contributor to systemic infection of intraperitoneally infected chickens}

Passing the intestines and establishing a systemic infection are two discrete steps in the pathogenicity of Salmonella serovars (Mastroeni and Grant, 2011). To further elucidate the role of polyamines in the systemic phase of the disease we tested the biosynthesis mutants by intra peritoneal (I.P.) infections of chickens (Table 4). Interestingly, using this route of infection, the $\Delta s p e B$-mutant vs the

Table 4

Competitive index analyses of $S$. Gallinarum mutants in intraperitoneal infection.

\begin{tabular}{lll}
\hline Mutant vs wt & C.I \pm S.D. & Statistical analyses \\
\hline$\Delta$ spe $B$ & $0.98 \pm 0.67$ Vs 1.0, \\
& & not significant \\
& & \\
$\Delta$ speE & $0.28 \pm 0.08$ & Vs $1.0, p<0.00001$ \\
$\Delta$ spe $; \Delta$ speE & $0.20 \pm 0.06$ & Vs $1.0, p<0.00001$ \\
$\Delta$ speB; $\Delta$ speE/pACYC-speB & $0.41 \pm 0.18$ Vs $\Delta s p e E$, not significant \\
$\Delta$ potCD & $0.18 \pm 0.21$ Vs $1.0, p=0.008$ \\
$\Delta$ potCD; $\Delta$ speE & $0.04 \pm 0.04$ Vs $1.0, p<0.00001$ \\
$\Delta$ potCD; $\Delta$ speE/pACYC-potCD & $0.21 \pm 0.13$ Vs $\Delta s p e E$, not significant \\
\hline
\end{tabular}

Infections were performed as described in Section 2. Competitive indexes (C.I.) were calculated as described in Section 2. Standard deviation (S.D.) of the mean is indicated. Statistical analyses were performed as described in Section 2. wt had a C.I. close to $1(0.98 \pm 0.67)$, indicating that putrescine biosynthesis is less important for the systemic phase of the disease. In contrast, the $\Delta$ speE-mutant had a C.I. of $0.28 \pm 0.08$ indicating that spermidine biosynthesis does contribute to virulence. The additional deletion of the $s p e B$ gene in this mutant only had a minor effect on the C.I. ( $\triangle$ speB; $\Delta$ speE C.I. $=0.2 \pm 0.06$ ), supporting the observation that putrescine biosynthesis has a limited role in the systemic phase of the disease.

The $\Delta$ speE-mutant can be expected to be only partially reduced in cellular spermidine concentrations as it retains the ability to take up available spermidine from the host environment through the PotABCD spermidine transporter. We therefore constructed a $\triangle$ pot $C D$-mutant and a $\triangle$ speE; $\triangle$ potCD double mutant to further investigate the specific role of spermidine in virulence. The $\Delta$ speE; $\triangle$ pot $C D$ double mutant is unable to synthesize and import spermidine, yet retains the ability to import and synthesize putrescine. The $\triangle$ pot $C D$-mutant had a C.I. of $0.18 \pm 0.21$, slightly lower than the $\triangle$ speE-mutant, however the $\triangle$ speE; $\triangle$ potCD double mutant was severely reduced with a C.I. of $0.04 \pm 0.04$. Virulence could be restored back to the $\triangle$ speE-mutant level by re-introducing the potCD genes on a plasmid. Together, our results strongly indicate that spermidine is essential for systemic infections of chickens by $S$. Gallinarum and that putrescine is less important for the systemic niche. One could argue that attenuation of the speEmutant could be caused by either toxic accumulation of putrescine inside the bacteria, or by reduced access to putrescine in the host environment. However, several of our results argue against these possibilities. Firstly, if putrescine accumulated to toxic levels inside the speE-mutant causing its reduced virulence it should not further reduce its virulence by disrupting the spermidine transporter PotABCD. Secondly, the bacteria have a putrescine exporter encoded by the potE-gene. This transporter would likely counter the effect of excess intracellular putrescine by pumping it out. Thirdly, if putrescine and spermidine are equally important for systemic infection, we would expect to have a reduced virulence for both the $s p e B$ - and the speE-mutants. Rather, the lack of a phenotype for the speB-mutant indicates that there is sufficient putrescine in the environment to feed into spermidine biosynthesis by SpeE, but this putrescine does not complement the reduced virulence of the speE-mutant. We therefore conclude that spermidine is more important than putrescine for the systemic phase of fowl typhoid.

\subsection{Polyamines are not required for intracellular survival and replication of $S$. Gallinarum in cultured HD-11 chicken macrophages}

A central feature of Salmonella infections is the ability of Salmonella to survive and replicate in host cells inside a specialized compartment termed the Salmonella containing vacuole (SCV) (Mastroeni and Grant, 2011). To investigate if the reduced virulence of the spermidine mutant was linked to defects in survival inside host cells, intracellular survival and replication of the polyamine mutants was assessed by enumerating intracellular bacteria $2 \mathrm{~h}, 4 \mathrm{~h}$ and $24 \mathrm{~h}$ post-infection of HD-11 chicken macrophages. In this assay, all strains had a similar 


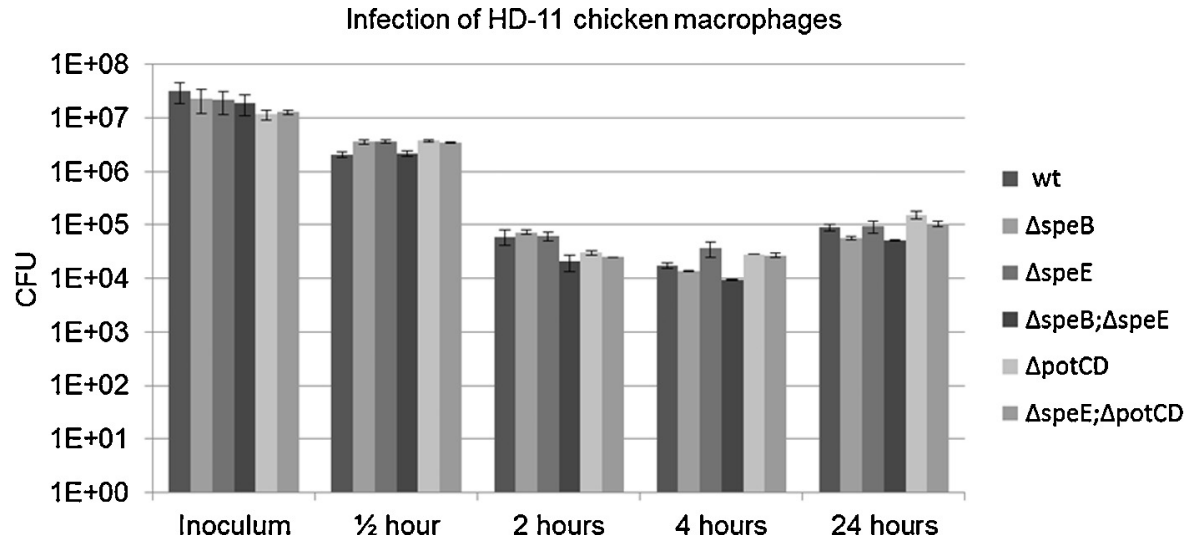

Fig. 2. Intracellular survival of $S$. Gallinarum strains in HD-11 chicken macrophages. HD-11 chicken macrophages were infected with the indicated strains of $S$. Gallinarum as described in Section 2. Bacteria were added to monolayers of HD-11 macrophages and incubated for 30 min. At this point gentamicin was added to the cell-cultures to kill extracellular bacteria. At the indicated time-points, cfu in the wells was measured.

approximate three-to-five fold net-replication between $4 \mathrm{~h}$ and $24 \mathrm{~h}$ p.i. The level of replication for the wt observed in this assay is similar to the level others have reported (Blondel et al., 2013). In contrast to observation in $S$. Typhimurium (Jelsbak et al., 2012), these results indicate that intracellular replication of $S$. Gallinarum is not affected by polyamines (Fig. 2). The reason for this discrepancy is not known, however, in S. Typhimurium intracellular replication was investigated in epithelial cells and only for the first $8 \mathrm{~h}$ p.i. perhaps resulting in different results. Alternatively, the specific roles of polyamines in virulence of the two serovars may be diverse, reflecting their diverse disease manifestations in their respective hosts.

\section{Concluding remarks}

In the present work we show that polyamines are essential for the host-restricted serovar S. Gallinarum despite evolutionary loss of some polyamine biosynthesis pathways. These results support the hypothesis that the extensive genome reduction observed in these serovars reflects the narrow niche they occupy where they have lost redundant/excess metabolic pathways compared to the metabolically robust serovars $S$. Typhimurium and $S$. Enteritidis that thrive in diverse environments. Additionally, our results extend recent reports that polyamines are essential for or contribute to virulence of several intracellular pathogens, like S. Typhimurium, Legionella pneumophila, Shigella spp., and Francisella tularensis (Carlson, Jr. et al., 2009; Russo et al., 2011; Nasrallah et al., 2011; Barbagallo et al., 2011; Jelsbak et al., 2012). The accumulating evidence on the role of polyamines in virulence of several intracellular pathogens suggests that polyamine biosynthesis could be attractive targets for development of novel anti-infective drugs for treatment of infections by a variety of intracellular bacteria.

It is interesting to note that in S. Gallinarum putrescine and spermidine appears to have different roles with spermidine being most important for the systemic phase of the disease. The reasons for this are not clear, however, spermidine has three amine-groups whereas putrescine only has two. This results in different charges with spermidine having one more positive charge than putrescine thereby affecting the interactions with other molecules of the cell. This could result in distinct physiological roles for the two polyamines. Accordingly, in Vibrio cholerae, it has been shown that exogenous spermidine and putrescine have opposite effects on expression of the Type IV pili (Goforth et al., 2013), and in Bacillus subtilis only the polyamine norspermidine is a signal for disassembly of biofilms (Kolodkin-Gal et al., 2012). Together with our results, these studies underscore the distinct roles different polyamines may have in bacterial physiology.

\section{Acknowledgements}

This work was funded by the Danish Research Council for Technology and Production through Grant Nos. 274-070328 and 12-126640. Casper Schroll was supported by a $\mathrm{PhD}$ stipend jointly financed by the Research School for Biotechnology, University of Copenhagen and the Technical University of Denmark.

\section{References}

Altschul, S.F., Madden, T.L., Schaffer, A.A., Zhang, J., Zhang, Z., Miller, W., Lipman, D.J., 1997. Gapped BLAST and PSI-BLAST: a new generation of protein database search programs. Nucleic Acids Res. 25, 3389-3402.

Barbagallo, M., Di Martino, M.L., Marcocci, L., Pietrangeli, P., De, C.E., Casalino, M., et al., 2011. A new piece of the shigella pathogenicity puzzle: spermidine accumulationby silencing of the speG gene. PLoS ONE 6, e27226.

Benson, D.A., Karsch-Mizrachi, I., Clark, K., Lipman, D.J., Ostell, J., Sayers, E.W., 2012. GenBank. Nucleic Acids Res. 40, D48-D53.

Blondel, C.J., Yang, H.J., Castro, B., Chiang, S., Toro, C.S., Zaldivar, M., et al., 2010. Contribution of the type VI secretion system encoded in SPI-19 to chicken colonization by Salmonella enterica serotypes Gallinarum and Enteritidis. PLoS ONE 5, e11724.

Blondel, C.J., Jimenez, J.C., Leiva, L.E., Alvarez, S.A., Pinto, B.I., Contreras, F., et al., 2013. The type VI secretion system encoded in Salmonella pathogenicity island 19 is required for Salmonella enterica serotype Gallinarum survival within infected macrophages. Infect. Immun. 81, 1207-1220.

Carlson Jr., P.E., Horzempa, J., O’Dee, D.M., Robinson, C.M., Neophytou, P., Labrinidis, A., Nau, G.J., 2009. Global transcriptional response to spermine, a component of the intramacrophage environment, reveals regulation of Francisella gene expression through insertion sequence elements. J. Bacteriol. 191, 6855-6864. 
Chadfield, M.S., Brown, D.J., Aabo, S., Christensen, J.P., Olsen, J.E., 2003. Comparison of intestinal invasion and macrophage response of Salmonella Gallinarum and other host-adapted Salmonella enterica serovars in the avian host. Vet. Microbiol. 92, 49-64.

Chang, A.C., Cohen, S.N., 1978. Construction and characterization of amplifiable multicopy DNA cloning vehicles derived from the P15A cryptic miniplasmid. J. Bacteriol. 134, 1141-1156.

Crichton, P.B., Old, D.C., 1990. Salmonellae of serotypes gallinarum and pullorum grouped by biotyping and fimbrial-gene probing. J. Med. Microbiol. 32, 145-152.

Datsenko, K.A., Wanner, B.L., 2000. One step inactivation of chromosomal genes in Escherichia coli K-12 using PCR products. Proc. Natl. Acad. Sci. U. S. A. 97, 6640-6645.

Feng, Y., Johnston, R.N., Liu, G.R., Liu, S.L., 2013. Genomic comparison between Salmonella Gallinarum and Pullorum: differential pseudogene formation under common host restriction. PLOS ONE 8 , e59427.

Goforth, J.B., Walter, N.E., Karatan, E., 2013. Effects of polyamines on Vibrio cholerae virulence properties. PLoS ONE 8, e60765.

Holt, K.E., Thomson, N.R., Wain, J., Langridge, G.C., Hasan, R., Bhutta, Z.A., et al., 2009. Pseudogene accumulation in the evolutionary histories of Salmonella enterica serovars Paratyphi A and Typhi. BMC Genomics $10,36$.

Igarashi, K., Kashiwagi, K., 2009. Modulation of cellular function by polyamines. Int. J. Biochem. Cell Biol..

Jelsbak, L., Thomsen, L.E., Wallrodt, I., Jensen, P.R., Olsen, J.E., 2012. Polyamines are required for virulence in Salmonella enterica serovar Typhimurium. PLOS ONE 7, e36149.

Jones, M.A., Wigley, P., Page, K.L., Hulme, S.D., Barrow, P.A., 2001. Salmonella enterica serovar Gallinarum requires the Salmonella pathogenicity island 2 type III secretion system but not the Salmonella pathogenicity island 1 type III secretion system for virulence in chickens. Infect. Immun. 69, 5471-5476.

Kaniga, K., Trollinger, D., Galan, J.E., 1995. Identification of two targets of the type III protein secretion system encoded by the inv and spa loci of Salmonella Typhimurium that have homology to the Shigella IpaD and IpaA proteins. J. Bacteriol. 177, 7078-7085

Kaniga, K., Tucker, S., Trollinger, D., Galan, J.E., 1995. Homologs of the Shigella IpaB and IpaC invasins are required for Salmonella Typhimurium entry into cultured epithelial cells. J. Bacteriol. 177 3965-3971.
Knodler, L.A., Vallance, B.A., Celli, J., Winfree, S., Hansen, B., Montero, M. Steele-Mortimer, O., 2010. Dissemination of invasive Salmonella via bacterial-induced extrusion of mucosal epithelia. Proc. Natl. Acad. Sci. U. S. A..

Kolodkin-Gal, I., Cao, S., Chai, L., Bottcher, T., Kolter, R., Clardy, J., Losick, R., 2012. A self-produced trigger for biofilm disassembly that targets exopolysaccharide. Cell 149, 684-692.

Larkin, M.A., Blackshields, G., Brown, N.P., Chenna, R., McGettigan, P.A McWilliam, H., et al., 2007. Clustal $W$ and Clustal X version 2.0. Bioinformatics 23, 2947-2948.

Mastroeni, P., Grant, A.J., 2011. Spread of Salmonella enterica in the body during systemic infection: unravelling host and pathogen determinants. Expert Rev. Mol. Med. 13, e12.

Nasrallah, G.K., Riveroll, A.L., Chong, A., Murray, L.E., Lewis, P.J., Garduno, R.A., 2011. Legionella pneumophila requires polyamines for optimal intracellular growth. J. Bacteriol. 193, 4346-4360.

Olsen, J.E., Hoegh-Andersen, K.H., Rosenkrantz, J.T., Schroll, C., Casadesus, J., Aabo, S., Christensen, J.P., 2013. Intestinal invasion of Salmonella enterica serovar Typhimurium in the avian host is dose dependent and does not depend on motility and chemotaxis. Vet. Microbiol.

Parkhill, J., Dougan, G., James, K.D., Thomson, N.R., Pickard, D., Wain, J., et al., 2001. Complete genome sequence of a multiple drug resistant Salmonella enterica serovar Typhi CT18. Nature 413, 848-852.

Rice, P., Longden, I., Bleasby, A., 2000. EMBOSS: the European Molecular Biology Open Software Suite. Trends Genet. 16, 276-277.

Russo, B.C., Horzempa, J., O’Dee, D.M., Schmitt, D.M., Brown, M.J., Carlson Jr., P.E., et al., 2011. A Francisella tularensis locus required for spermine responsiveness is necessary for virulence. Infect. Immun..

Thomson, N.R., Clayton, D.J., Windhorst, D., Vernikos, G., Davidson, S. Churcher, C., et al., 2008. Comparative genome analysis of Salmonella Enteritidis PT4 and Salmonella Gallinarum 287/91 provides insights into evolutionary and host adaptation pathways. Genome Res. 18, 1624-1637.

Uzzau, S., Leori, G.S., Petruzzi, V., Watson, P.R., Schianchi, G., Bacciu, D., et al., 2001. Salmonella enterica serovar-host specificity does not correlate with the magnitude of intestinal invasion in sheep. Infect. Immun. 69, 3092-3099.

Wallrodt, I., Jelsbak, L., Thorndahl, L., Thomsen, L.E., Lemire, S., Olsen, J.E., 2013. The putative thiosulfate sulfurtransferases PspE and GlpE contribute to virulence of Salmonella Typhimurium in the mouse model of systemic disease. PLoS ONE 8, e70829. 